\title{
Anticipatory Movement Timing Using Prediction and External Cues
}

\author{
Jeremy B. Badler and Stephen J. Heinen \\ The Smith-Kettlewell Eye Research Institute, San Francisco, California 94115
}

\begin{abstract}
Animals often make anticipatory movements to compensate for slow reaction times. Anticipatory movements can be timed using external, sensory cues, or by an internal prediction of when an event will occur. However, it is unknown whether external or internal cues dominate the anticipatory response when both are present. Smooth pursuit eye movements are generated by a motor system heavily influenced by anticipation. We measured pursuit to determine how its timing was influenced when both a predictable event and a visual cue were present. Monkeys tracked a moving target that appeared at a constant time relative to the onset of a fixation point. At a randomized time before target onset, the fixation point disappeared, creating a temporal "gap" that cued impending target motion. We found that the gap onset cue and prediction of target onset together determined pursuit initiation time. We also investigated whether prediction could override the gap onset cue or vice versa by manipulating target onset and, hence, the duration of time that the animal had to estimate to predict it. When target motion began earlier, the pursuit system relied more on prediction to trigger a movement, whereas the cue was more often used when the target moved later. Pursuit latency in previous trials partially accounted for this behavior. The results suggest that neither internal nor external factors dominate to control the anticipatory response and that the relative contributions vary with stimulus conditions. A model in which neuronal anticipation and fixation signals interact can explain the results.
\end{abstract}

Key words: smooth pursuit; eye movements; gap; expectation; interaction; primate

\section{Introduction}

For survival in the wild, it is critical that an animal perform accurate and rapid motor actions in response to external events. However, in primates and other mammals, delays inherent in the sensory systems that detect those events can compromise response times. To compensate for sensory delays, these animals have evolved the capability to make anticipatory movements. Anticipatory movements are generated internally, and are based on a prediction of the time that they would be appropriate. However, it is not always possible to predict when an event will occur, in which case an external stimulus, or cue, can trigger the response. For example, in a predator/prey situation, subtle gestures might signal that an attack or flight will occur. When cues are reliable, they can override or replace internal predictions (Barnes and Donelan, 1999). However, cues in nature are not always reliable. In such situations, the animal must decide whether to use the cue or fall back on prediction.

The aim of the current study was to determine the relative contributions of an unreliable cue and a predictable target to the timing of movement initiation. To this end, we measured smooth pursuit eye movements in a task where an unreliable cue preceded the onset of a moving target, the timing of which was

Received Sept. 3, 2005; revised March 16, 2006; accepted March 17, 2006.

This work was supported by National Institutes of Health Grant R01-EY11720. We thank M. Missal for assistance with data collection, and the members of the S. J. Heinen and E. L. Keller laboratories for helpful comments on this manuscript.

Correspondence should be addressed to Stephen J. Heinen, The Smith-Kettlewell Eye Research Institute, 2318 Fillmore Street, San Francisco, CA 94115. E-mail: heinen@ski.org.

D01:10.1523/JNEUROSCI.3739-05.2006

Copyright $\odot 2006$ Society for Neuroscience $\quad$ 0270-6474/06/264519-07\$15.00/0 always predictable. As a cue, a visible fixation point was extinguished a variable amount of time before the target moved. Prediction difficulty was manipulated separately by changing the total amount of time before target appearance, because the timing of a long interval can be estimated with less precision than that of a short interval (Gibbon et al., 1997).

Anticipation is a pervasive aspect of behavior (Kowler, 1989; Witney et al., 2000; Wexler and Klam, 2001). A motor system in which the role of anticipation has been extensively studied is the smooth pursuit eye-movement system. Although the pursuit system responds to the motion of an object (Lisberger et al., 1987; Keller and Heinen, 1991), pursuit behavior is influenced heavily by the anticipation of future target motion in humans (Kowler, 1990; Heinen and Keller, 2003). Nonhuman primates can also use smooth pursuit to anticipate the onset of a moving target (Missal and Heinen, 2004). Anticipation is enhanced when targets are predictable (Kowler et al., 1984; Kowler, 1989; Barnes and Asselman, 1991; Kao and Morrow, 1994; Barnes and Donelan, 1999; Heinen et al., 2005), when targets move at high speeds (Kao and Morrow, 1994; Heinen et al., 2005), and when a time delay ("gap") is present between fixation point disappearance and target appearance (Boman and Hotson, 1988). Anticipatory pursuit is also triggered by sensory cues that reliably precede target motion onset, even when the onset itself is not predictable (Kowler, 1989; Barnes and Donelan, 1999).

When we measured anticipatory pursuit in the presence of an unreliable gap cue and predictable target onset time, we found that both factors contributed, with reliance on the cue increasing as the target became more difficult to predict. The results suggest that anticipatory pursuit initiation depends on both in-trial cues 


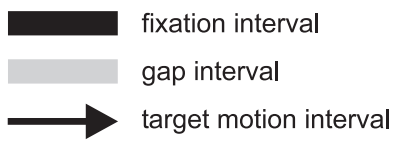

A

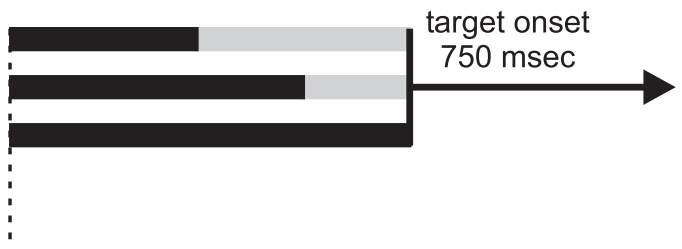

B

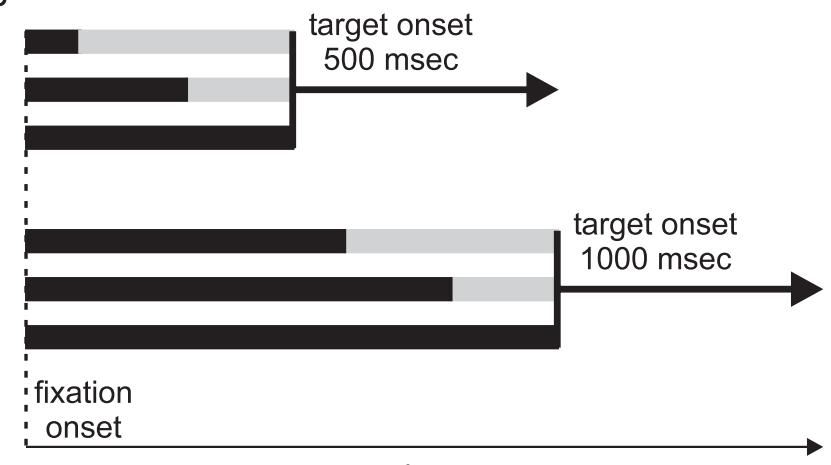

time

Figure 1. Experimental paradigm. $A$, Block design of the first experiment, showing three of the 10 possible fixation/gap configurations. Target onset time was constant at $750 \mathrm{~ms}$. Gap duration varied from 0 to $450 \mathrm{~ms}$, and fixation duration was equal to target onset minus gap duration. $\boldsymbol{B}$, Additional block designs used in the second experiment. Target onset within a block was either $500 \mathrm{~ms}$ (top) or $1000 \mathrm{~ms}$ (bottom), with possible gap durations unchanged. For all experiments, pursuit latencies were measured with respect to the onset of the fixation interval (dashed line).

and predictions about upcoming stimuli. We propose a conceptual model in which anticipatory pursuit is controlled by interactions of known fixation and anticipation processes in the brain. These results have been presented previously in preliminary form.

\section{Materials and Methods}

All procedures were approved by the California Pacific Medical Center Institutional Animal Care and Use Committee. Head holders and ocular search coils were surgically implanted in two juvenile male macaque monkeys (BU and SA) as described previously (Missal and Heinen, 2001). Monkeys were seated in a darkened room facing a dimly lighted tangent screen. The fixation period began when a $1^{\circ}$ spot of light from a projection oscilloscope was illuminated in the center of the screen, after a tone. After bringing its gaze to the spot, the animal was required to continue to fixate until the appearance of a target spot. On gap trials, the fixation spot disappeared at a variable time before target onset (Fig. 1). After fixation, the central spot reappeared (on gap trials) and moved always in the same direction on each trial within a block (right for monkey SA; left for monkey BU) and at the same speed (50 deg/s). During the fixation and gap intervals, the monkey was required to hold its gaze in a $10^{\circ}$ square electronic window around the fixation point. The large window was used so as not to penalize the monkey for anticipatory movements which would have exceeded a smaller window. When the pursuit target appeared, the monkey had to track it within a $3^{\circ}$ window. Liquid reward was given after successfully tracking the target across $25^{\circ}$ of visual angle. Experiments were controlled using software developed in the lab.

Trials were organized into blocks within which target onset time was held constant and fixation and gap durations covaried (Fig. 1). Three target onset times were used. In the first experiment (mid-range block) (Fig. 1A), target onset was set equal to $750 \mathrm{~ms}$, relative to the onset of the

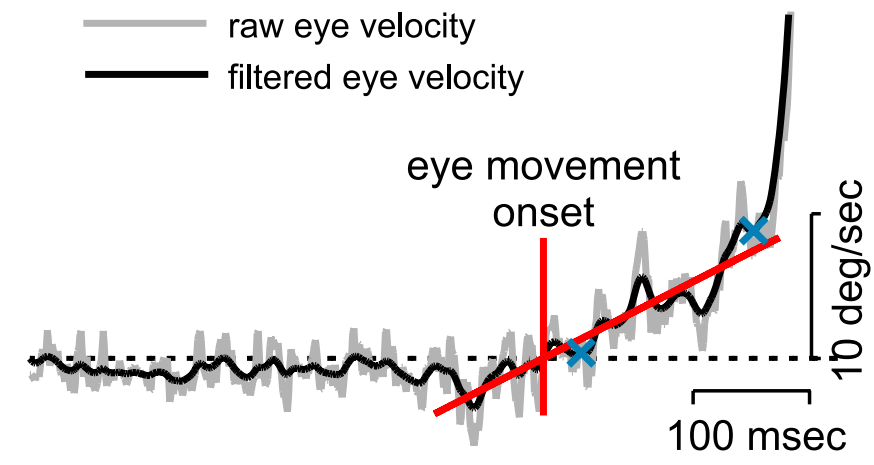

Figure 2. Interpolation method used to detect anticipatory pursuit. The blue $\mathrm{Xs}$ are userselected points on the filtered eye trace, and the sloped red line is the resulting regression fit. The short vertical line indicates where the regression line intersects the zero-velocity baseline (dotted line), and is defined as eye-movement onset.

fixation interval. Fixation intervals ranged from $300-750 \mathrm{~ms}$, in increments of $50 \mathrm{~ms}$. The gap intervals filled the time voids before target onset, ranging from $450 \mathrm{~ms}$ (300 ms fixation) to $0 \mathrm{~ms}$ ( $750 \mathrm{~ms}$ fixation). Hence, the terms "fixation point disappearance" and "gap onset" are used interchangeably. The second experiment (Fig. $1 B$ ) was set up similarly, but used two types of trial blocks. The early target blocks had a target onset time of $500 \mathrm{~ms}$. Fixation durations ranged from $50-500 \mathrm{~ms}$ for monkey $\mathrm{BU}$ and $100-500 \mathrm{~ms}$ for monkey SA, leaving gap durations ranging from 450 to 0 and 400 to $0 \mathrm{~ms}$ in the two monkeys, respectively. The late-target blocks had target onset equal to $1000 \mathrm{~ms}$, with fixation durations ranging from 550 to $1000 \mathrm{~ms}$, and gap durations ranging from 450 to $0 \mathrm{~ms}$ for both monkeys. Each block typically consisted of 50-100 trials, and several blocks of the same type were always run in succession. Before each block series, the monkey was given $20-50$ practice trials for that particular block type, which were not recorded.

Eye position was digitized at $1000 \mathrm{~Hz}$ using an analog-to-digital converter (National Instruments, Austin, TX). Analysis was done offline using Matlab (Mathworks, Natick, MA) on a PC system. Eye position was digitally differentiated and filtered with a noncausal two-pole Butterworth filter (cutoff $25 \mathrm{~Hz}$ ) to obtain eye velocity. Anticipatory pursuit velocity was computed by averaging eye velocity in a $20 \mathrm{~ms}$ bin centered on target onset, as has been done previously (Missal and Heinen, 2004). Pursuit onset time was determined using an interpolation algorithm similar to that used by previous investigators (Carl and Gellman, 1987; Krauzlis and Miles, 1996). Briefly, our algorithm was as follows (Fig. 2). An operator selected two points on an approximately linear section of the horizontal eye velocity trace, which defined the range over which a linear regression line was constructed. Pursuit onset was defined as the time when the fit line intersected zero. Note that if anticipatory pursuit per se is absent from a trace, this method simply detects visual pursuit onset. Because we did not wish to impose an artificial acceleration or latency criterion to separate anticipatory pursuit onset from visually guided pursuit onset, we used the entire data set and refer to pursuit onset and anticipatory pursuit onset interchangeably.

\section{Results}

A total of 3922 trials were recorded from monkey BU and 3895 trials from monkey SA. Trials in which the monkeys failed to track the target (BU: 276, 7.0\%; SA:317, 8.1\%) were not included. Trials in which saccades interfered with anticipatory pursuit onset, or drowsiness-related slow eye movements contaminated the record were also eliminated (BU: 272, 6.9\%; SA: 393, 10.1\%). Trials in which saccades contaminated the $20 \mathrm{~ms}$ time bin centered at target onset (BU: 161, 4.1\%; SA: 143, 3.7\%) were excluded from the anticipatory velocity analysis only.

Typical examples of observed pursuit initiation behavior are shown in Figure 3. The most common pattern is shown in Figure $3 A$, where robust anticipatory pursuit developed during the gap 


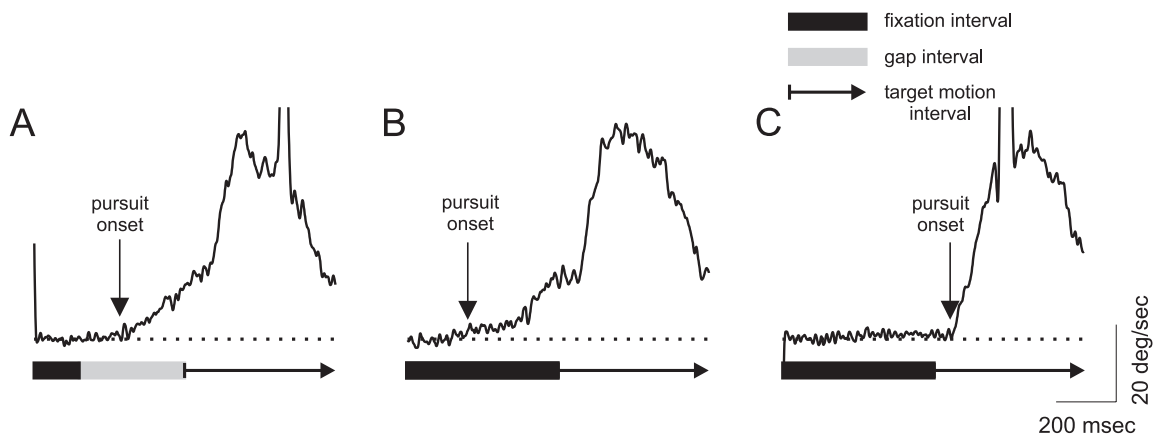

Figure 3. Typical pursuit traces. $\boldsymbol{A}$, Anticipatory pursuit began during the gap interval. $\boldsymbol{B}$, In a no-gap trial anticipatory pursuit began during the fixation interval. Note that the need for a catch-up saccade was mitigated. $\boldsymbol{C}$, Not all trials showed anticipatory pursuit. For all panels, only part of the fixation and target motion intervals are shown.

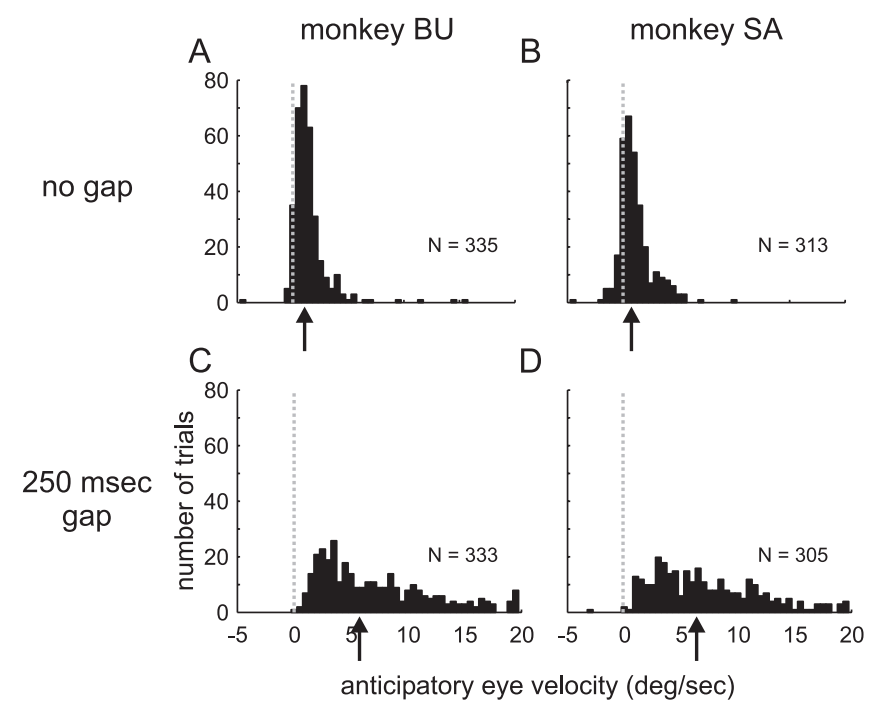

Figure 4. Histograms of anticipatory pursuit velocity. $\boldsymbol{A}, \boldsymbol{B}$, In both monkeys, anticipation occurred in the no-gap condition. $\boldsymbol{C}, \boldsymbol{D}$, Anticipation was more frequent when there was a gap, and also reached higher velocities. The total number of trials are noted and arrows indicate median values. Data for monkey BU are on the left and data for SA are on the right.

interval. Pursuit sometimes began during the fixation interval, especially if the gap was short or absent (Fig. 3B). Finally, in some trials (usually no-gap) no anticipatory pursuit was generated (Fig. 3C).

A gap has been shown to enhance human anticipatory pursuit velocity (Boman and Hotson, 1988). We first investigated whether this was also the case for monkeys, and found that it was. Figure 4 shows frequency histograms of eye velocity for the nogap $(A, B)$ and $250 \mathrm{~ms}$ gap $(C, D)$ conditions for the two monkeys. When there was no gap, anticipatory velocity was significantly higher than zero (sign test, $p<0.0001$ for both monkeys). In the presence of a gap, anticipatory velocity was significantly higher than in the no-gap condition (Wilcoxon rank-sum test, $p<$ 0.0001 for both monkeys). The difference was significant for all other gap durations tested except $50 \mathrm{~ms}$. Thus, the presence of a gap can facilitate anticipatory pursuit in monkeys, although it is not necessary to evoke it.

Our primary question was how prediction and cuing both contribute to determine pursuit timing. To this end, we placed the animal in a situation where it could use either information about the time that the target moved or the disappearance of the fixation point to generate anticipatory pursuit. We found that both target and gap onset times contributed to pursuit onset time. Pursuit onset versus gap are plotted for monkey BU in Figure 5, for all blocks of trials in which target onset $=750 \mathrm{~ms}$. The distribution of onset times shown here is not a simple function of either factor. Pursuit usually began during the gap interval (denoted by the gray triangle). Most onset time points lie parallel to the lower boundary of the triangle, which corresponds to gap onset. Furthermore, many of these points appear to occur $\sim 100 \mathrm{~ms}$ after this time. However, for early gap onsets, there are some trials where pursuit began late in the gap interval, and for late gap onsets, pursuit sometimes began before the gap interval. Finally, note that there are points that lie $80 \mathrm{~ms}$ or more above the triangle. These represent trials in which anticipatory pursuit was absent and the first pursuit movement was visually guided after the onset of target motion.

To quantify these data, we first considered the two extreme hypotheses about what the animal was using to generate anticipatory pursuit. If the monkey was only predicting the time that the target moved and was ignoring the cue, the resulting pursuit onset time points would be distributed parallel to the line of target onset time. Equivalently, a linear regression performed on the onset times would yield a line of zero slope. Note that this line is expected to lead target onset, to allow the monkey enough time to accelerate and match target velocity. It is schematized by the line labeled "predict only" in Figure 5. The other extreme is that the animal simply used the disappearance of the fixation point as a cue to initiate pursuit on every trial, yielding a distribution of onset time points that lie parallel to the line of gap onset time. A regression would yield a line of unity slope, such as the one labeled "cue only" on the figure. The actual regression line is labeled "fit to data." Its slope was almost exactly between zero and one: 0.52 ( $\pm 0.05 \%, 95 \%$ confidence interval). The data for monkey SA (data not shown) had a similar slope $(0.41 \pm 0.05)$. This suggests that the monkeys used both strategies; for some trials they attempted to time their pursuit onset to target onset, and in others they simply began pursuit cued by the gap onset. Thus, pursuit initiation appears to depend on both a visual cue component and a predictive component under these conditions.

We next attempted to see whether we could bias the animals to rely more on a single strategy (prediction or cue response). Because short intervals of time can be estimated more reliably than long intervals (Gibbon et al., 1997), we varied, in different blocks, the amount of time before the target moved. The rationale was that when the interval between fixation onset and target onset is relatively short, the monkeys should be able to time their movements more accurately. Conversely, a long interval is expected to be more difficult to predict and, thus, the monkey would rely more on the gap onset cue. The experimental paradigm here was similar to the previous: gap durations ranged from $0-450 \mathrm{~ms}$ and target onset was constant in a trial block. However, in separate blocks, target onset time was set to either 500 or $1000 \mathrm{~ms}$. Because the gap onset cue always preceded target onset by the same range of times, the cue-response strategy remained equally reliable for all conditions.

Pursuit onset times from monkey BU for target onset $=500$ and target onset $=1000 \mathrm{~ms}$ are plotted in Figure $6, A$ and $B$, respectively. For the early target onset time $(500 \mathrm{~ms})$, the data points follow the prediction line more closely than they did for the $750 \mathrm{~ms}$ target (Fig. 5). Accordingly, the fit slope is shallower 


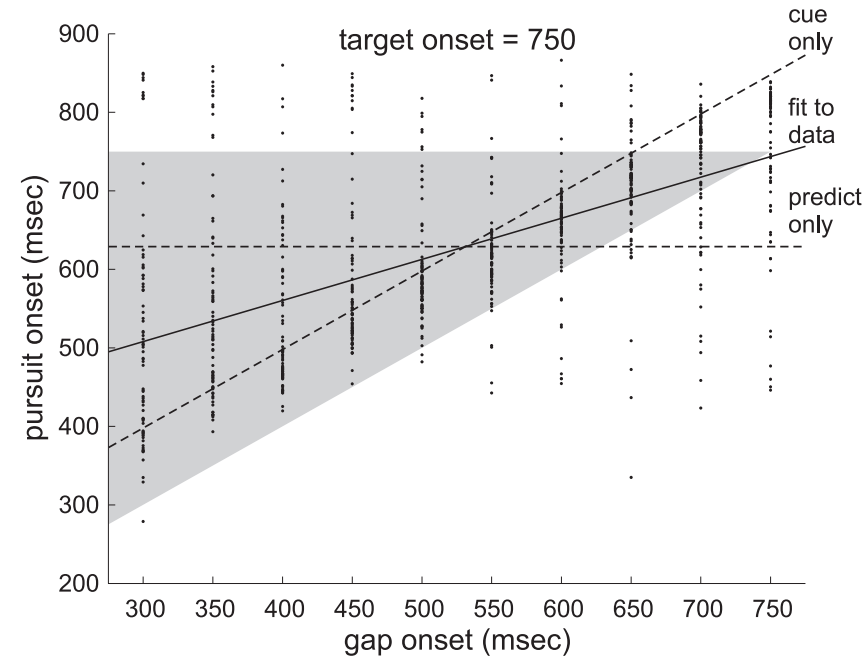

Figure 5. Pursuit onset times as a function of gap onset time, for monkey BU and target onset $=750 \mathrm{~ms}$. The gray triangle denotes the gap interval. The dashed line labeled "predict only" is a hypothetical regression fit with a slope equal to zero. It would result if the pursuitonset data were determined solely by an estimate of target onset time, ignoring the gap onset cue. The second dashed line, labeled "cue only," is another hypothetical regression, with a slope equal to one. It represents the case if pursuit onset occurred solely in response to the gap onset cue, with no attempt to predict target onset. The actual regression fit to the data (solid line, "fit to data") lies between these two extremes, indicating that both prediction of target and response to cue influenced behavior over the course of the experiment.

[BU: $0.40 \pm 0.04$; SA (data not shown): $0.11 \pm 0.03$ ]. Thus, the prediction strategy was favored more in this condition by both monkeys. The data for the late target onset time $(1000 \mathrm{~ms})$ show the opposite trend, as the points now cluster around the gap onset line. In this condition, the fit slope is steeper [BU: $0.82 \pm 0.04$; SA (data not shown): $0.75 \pm 0.05]$. Therefore, for the most part, the cue-response strategy was used in this condition.

The regression slopes for all conditions in both monkeys are summarized in Figure 7. Shallow slopes (close to zero) are evidence that the monkey used a prediction strategy, and steeper slopes (close to one) indicate a cue-response strategy. As the interval between fixation onset and target onset increases, so do the slopes of the regression lines. Within each monkey, both pairs of differences (500 vs $750 \mathrm{~ms}$ and 750 vs $1000 \mathrm{~ms}$ ) were significant ( $t$ test, $p$ values $\leq 0.01$ ). Therefore, the monkeys appeared to shift from a largely prediction-driven strategy to a cue-driven strategy for estimating when to move as trial conditions became less favorable for prediction. However, note that even for the extreme timing conditions, the fit slopes are still significantly different from zero and one ( $t$ test, $p$ values $<0.0001$ ). This indicates that neither strategy was used exclusively.

Previous work has shown that stimulus history influences behavior (Kowler et al., 1984; Kowler, 1989; McPeek et al., 1999; Krauzlis and Adler, 2001; Heinen et al., 2005), and we tested whether this effect could, in part, account for the behavior of the animals in our experiments. Trials were grouped according to whether they were preceded by an early gap onset $(-450,-400$, $-350 \mathrm{~ms}$, with respect to target onset) or a late gap onset $(-100$, $-50,0 \mathrm{~ms}$ ) in two previous trials. Trials where the previous gap value lay outside these ranges were not included in the analysis. Each monkey and target onset time was grouped separately, for a total of six data sets. For each set, a Wilcoxon rank-sum test was applied to the pursuit onset time grouped by the immediately preceding trial. Although there was some tendency for pursuit latency in trials preceded by early gap onsets to be shorter than in
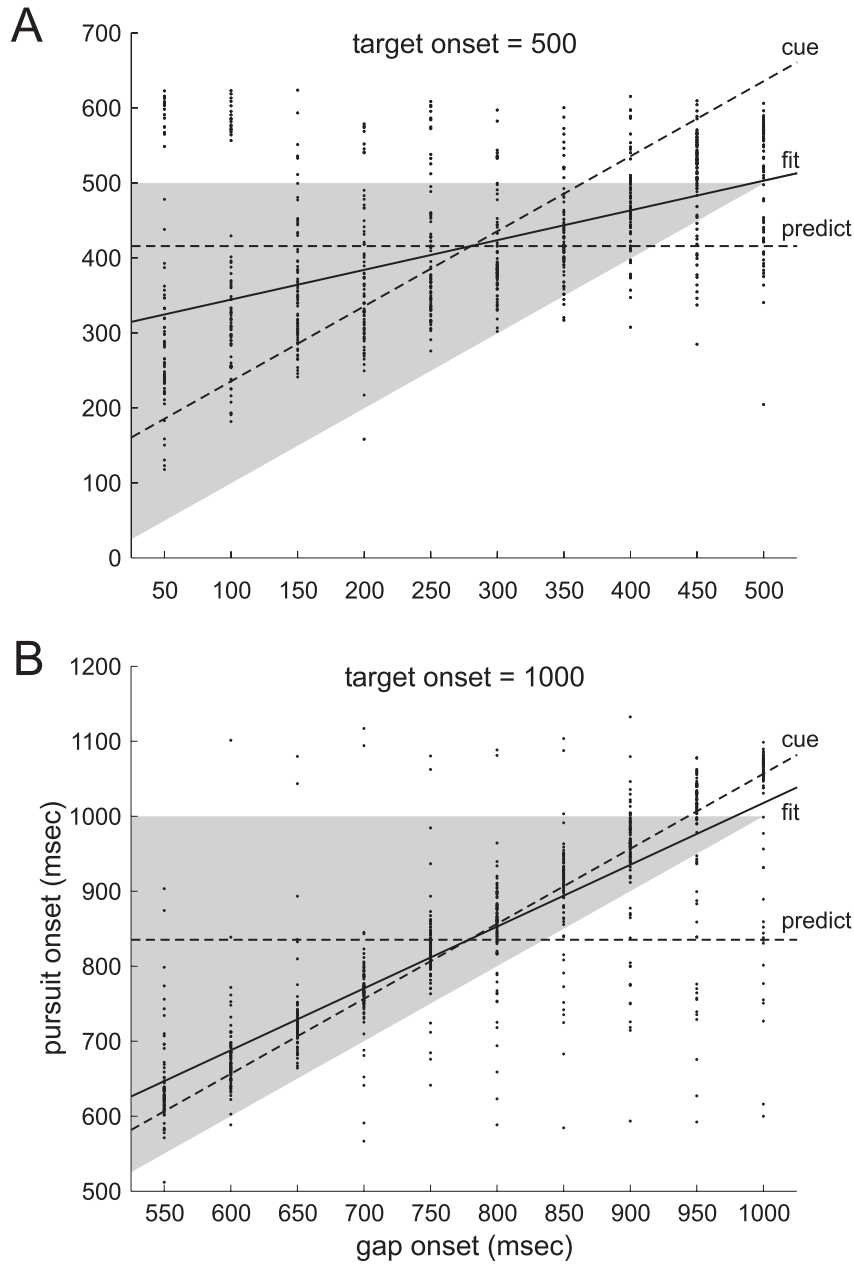

Figure 6. Pursuit onset times as a function of gap onset time. $A$, Data from blocks where target onset time $=500 \mathrm{~ms}$. B, Blocks where target onset $=1000 \mathrm{~ms}$. The monkey is BU. Other details are as in Figure 5 . Note that for the early target onset time, the slope of the pursuit-onset data are closer to zero, indicating a greater tendency toward prediction. For the late target onset time, the data slope is closer to one, indicating a greater dependence on the gap onset cue.

trials preceded by late gap onsets, this trend was not significant ( $p>0.15$ for all conditions). This suggests that stimulus history did not have a significant influence in this experiment.

There is also evidence that previous behavior can influence latency in saccade tasks (Paré and Munoz, 1996; Dorris et al., 1999). Given that stimulus history and behavioral history are likely correlated in our experiment, and the fact that we saw a trend suggestive of a stimulus history effect, we decided to determine whether previous behavior had an effect on our results. To test for this, we reanalyzed our pursuit onset times based on whether the monkey moved early or late in either of the two previous trials. An early movement was defined as one having an onset time in the lower 30th percentile of all trials, and a late movement in the upper 30th percentile. Again, data were analyzed separately by monkey and target onset time, and both the first and second previous trial had to be in the early or late category for inclusion. Results for two conditions are shown in Figure 8. Data are plotted in a tree format (Kowler et al., 1984; Heinen et al., 2005), where the rightmost node indicates the mean pursuit onset in all trials, the center nodes indicate means grouped according to the first previous trial, and the leftmost nodes indicate means further grouped by the second previous trial. These plots show that the monkeys tended to move earlier when their previ- 


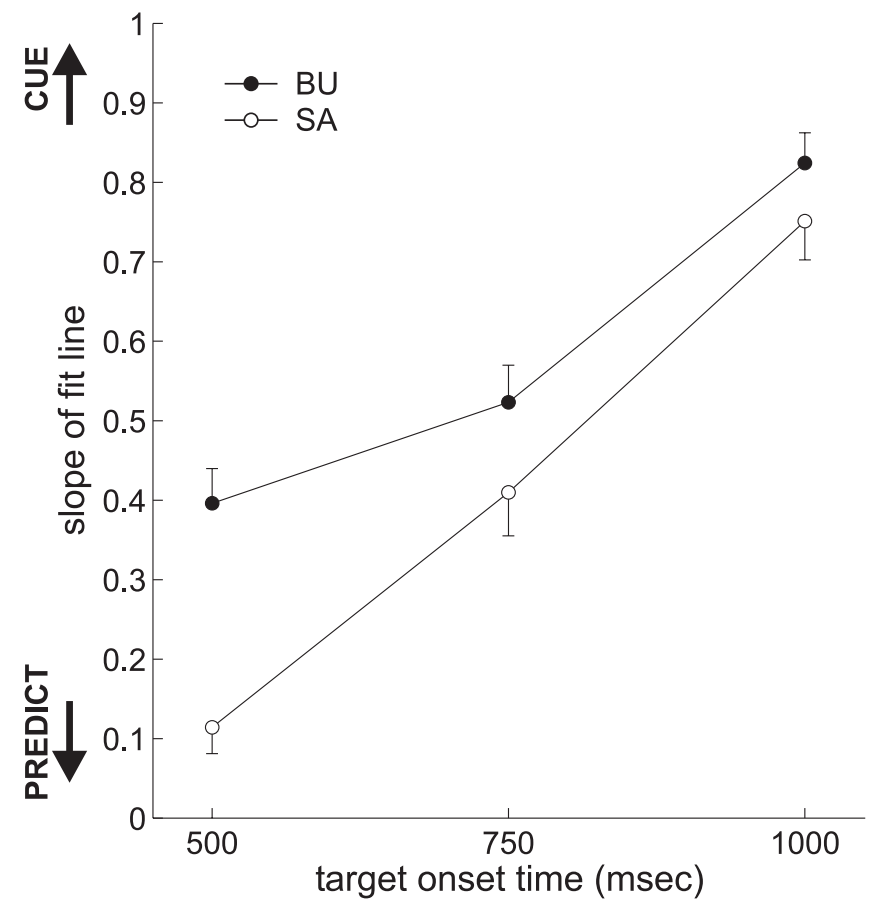

Figure 7. Regression slopes vary systematically with target onset time. For blocks with an early target onset, slopes are closer to zero, suggesting that prediction largely determines pursuit onset time. For blocks with a late target onset, slopes are closer to one, suggesting pursuit onset is more influenced by the visual cue of gap onset. Error bars are $95 \%$ confidence intervals.
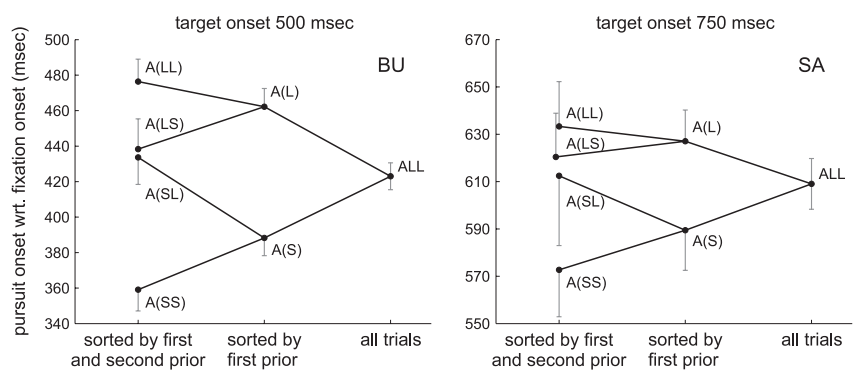

Figure 8. Effect of behavioral history on pursuit onset. Trials were grouped based on whether they were preceded by a short (S) or long (L) latency pursuit movement and plotted in a tree format. The rightmost nodes show mean pursuit onset for all qualifying trials. The middle nodes show the trials sorted by their immediately preceding trial, and the leftmost nodes further subdivide the data according to the two preceding trials. For example, the node labeled $A(L S)$ represents all trials preceded by a short latency movement that was in turn preceded by a long latency movement. Note that late pursuit trials tend to follow other late pursuit trials, and early pursuit trials tend to follow other early trials. Error bars represent SE. One node in the SA tree is offset slightly for clarity.

ous pursuit was earlier, and later when their previous pursuit was later. In five of six trees, the primary node pair was significantly different, and three secondary node pairs were significantly different as well (Table 1). Therefore, behavioral history was an additional factor that influenced pursuit onset.

\section{Discussion}

We found that the timing of anticipatory pursuit was a function of both a visual cue (the disappearance of a fixation point) and an expectation of a predictable target onset time, and was modulated by conditions in the set of trials being performed. When target onset time was earlier, relative to the start of the fixation interval, movement initiation times tended to predict the target onset event. When the target onset time was later, movements occurred more often in response to the beginning of the gap interval. The monkey's responses were also influenced by its behavior in previous trials in that early pursuit tended to follow other early pursuit trials, and late pursuit tended to follow late pursuit.

We also found that gap trials were associated with increased anticipatory eye velocity, consistent with the results of another study done with humans (Boman and Hotson, 1988). Anticipatory velocity was still significant in no-gap trials, showing that anticipation is a pervasive aspect of monkey smooth-pursuit behavior, as it is in humans (Kowler, 1990).

For visually guided saccades, there is evidence that the gap releases fixation mechanisms in the brain, allowing an earlier movement (Dorris and Munoz, 1995). Because there is evidence that smooth pursuit and saccades share a common fixation mechanism (Krauzlis and Miles, 1996; Missal and Keller, 2002), releasing that fixation mechanism may have been responsible for triggering anticipatory pursuit in trials that fit the cue-response strategy in our study. Indeed, there is evidence that using a cue reliably evokes anticipatory pursuit (Barnes and Donelan, 1999). However, in our paradigm, the cue is not a reliable predictor of target onset time because it precedes target onset by a variable amount. Thus, using the cue exclusively will lead to large tracking errors, because the eyes would move too soon for early gap onsets and too late for later gap onsets. An alternative strategy is to try and predict the timing of target onset. In this case, fixation would be released according to an internal time estimate, independently of the gap cue. If prediction were perfect on every trial, pursuit would begin at the optimal time for tracking the target. However, prediction is not entirely accurate, so there will still be tracking errors on individual trials. In addition, longer time intervals are more difficult to estimate than shorter ones (Gibbon et al., 1997), making prediction a less viable strategy under those conditions. This is reflected in Figures 6 and 7, where a smaller fraction of trials showed predictive behavior as target onset latency was increased.

One potential question is whether the gap is unique in its effect on anticipatory pursuit, or whether it is similar to any other warning cue. Our laboratory has performed preliminary experiments identical to those presented here, except where the gap has been replaced by a transient color change. Our results show two effects: first, anticipatory pursuit velocity was reduced, but not eliminated. Second, pursuit onset times showed less evidence of using the cue and as a result, reliance on prediction was increased. Thus, in our paradigm, the gap probably acts as an enhanced cue.

Our task is reminiscent of interval estimation tasks commonly used to study timing in the psychological literature, but it has several important differences. First, classical tasks use finger tapping or other motor responses from the subject (Collyer and Church, 1998). However, Collins et al. (1998) showed that principals of timing theory could be applied to repetitive eye movements. A second difference is that our task includes a gap onset cue, which could act as an attentional distractor that interferes with timing estimation (Casini and Macar, 1997). Moreover, the goal of our task is not to estimate an interval per se, but to track a moving spot. It is ultimately up to the animal whether it accomplishes this by estimating the target onset time or by using the gap onset cue, although experimental conditions sometimes favor one or the other approach.

The history of stimuli from previous trials has been shown to influence oculomotor behavior (Kowler et al., 1984; Kowler, 1989; McPeek et al., 1999; Krauzlis and Adler, 2001; Heinen et al., 2005). This is commonly attributed to a sensory priming mech- 
anism, whereby consecutive identical stimuli in previous trials could sensitize neural systems to respond more quickly to that stimulus (Maljkovic and Nakayama, 1994). In our study, we did not observe a significant effect of stimulus history, but rather, behavioral history because like behaviors (early or late movement) tended to follow each other. This has also been observed for saccade reaction times (Paré and Munoz, 1996; Dorris et al., 1999). We suspect our history result is a motor-priming effect analogous to sensory priming, where the pursuit system becomes calibrated to generate early or late movements on average, taking several trials before that calibration is adjusted.

To interpret our results, we turned to models of movement initiation. A prominent one is the race model (Logan et al., 1984), developed originally to simulate the distribution of reaction times in a countermanding paradigm, in which a subject needed to cancel a planned response at the appearance of a stop cue. The model was later applied to saccade countermanding tasks (Hanes and Schall, 1995). It consists of a movement-initiation process ("go") that competes against a movement-suppression process ("stop"), the outcome of which determines whether the animal will generate a saccade or not. The go and stop processes are traditionally treated as independent, although more recent studies have found evidence of an interaction between them (Ozyurt et al., 2003).

To describe anticipatory pursuit timing, we have formulated an alternative to the race model that is based more on physiology and does not assume independence. Our model (Fig. 9) consists of two interacting processes. The fixation component is responsible for inhibiting unwanted movements. This component is tonically active, but that activation is increased by the presence of a visible fixation point. Fixation component activity is similar to that of fixation cells found in the supplementary eye field (SEF) (Schlag and Schlag-Rey, 1987; Bon and Lucchetti, 1991; Schall, 1991; Schlag et al., 1992; Lee and Tehovnik, 1995), an area involved in anticipatory pursuit initiation (Missal and Heinen, 2004). The second model component is the anticipatory process. It acts as a timer, with activity that rises during the course of a trial. This process is based on activity recorded in SEF from preparatory or anticipation cells (Heinen and Liu, 1997). The fixation and anticipation components in the model interact in a mutually inhibitory manner. A similar interaction has been observed between fixation and movement cells in another oculomotor brain area, the superior colliculus (Munoz and Istvan, 1998).

In preliminary simulations of our model, the rate of rise of the anticipatory signal is increased or decreased by setting a "readiness" input. The steadily rising anticipation activity eventually suppresses the fixation activity, allowing a predictive movement to be triggered. Higher readiness values will accelerate this process, de-emphasizing the contribution of the fixation signal and generating a greater fraction of predictive movements. Lower readiness values have the opposite effect, facilitating cueresponse behavior by causing the system to be dominated by the fixation process. In this case, movements can only occur after the fixation point disappears, removing that source of inhibition. Thus, both behavioral variability within trial blocks and strategy changes between trial blocks can be simulated by changing the readiness value. In addition, by assuming that the readiness can only change by a limited amount between trials, we can simulate the history results where like behaviors tend to follow each other.

Another model of anticipatory pursuit initiation has been de-

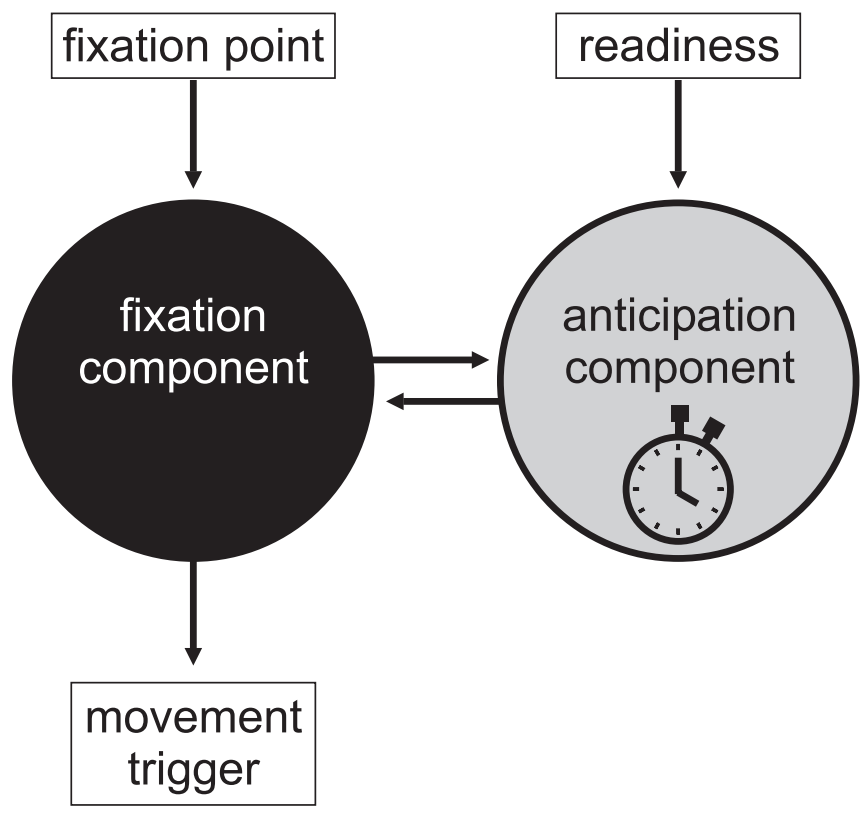

Figure 9. Conceptual model for pursuit initiation. The anticipation component acts as a clock that times expected target onset. The base clock rate is controlled by the readiness value. It varies randomly from trial to trial, as well as systematically according to the overall target onset time in a trial block. The fixation component responds to the onset of the gap interval and triggers the eye movement. There is an interaction between the fixation and anticipation components: if the anticipation clock is too fast, it will suppress the fixation mechanism and cause an early movement to be triggered. If the anticipation clock is slow, the fixation activity remains high, causing movements to be delayed.

veloped by Barnes et al. (1991). It contains a velocity storage element that is "charged" by exposure to repeated motion. The element then releases its charge as anticipatory eye velocity, with timing controlled by a periodicity estimator. Although designed to reproduce periodic tracking, the model could be modified and applied to a trial-based paradigm like ours, where the onset (and disappearance) of the fixation point cue impending target motion. Because the model requires several cycles to charge the predictor elements, it would not produce the anticipatory movements that we observe on the first few trials in a block. In addition, the neural implementation of the predictor elements has not been elaborated. Finally, to account for trials in which anticipation begins in response to the gap onset cue, the periodicity estimator must be deactivated or ignored. Our own model is one possible implementation of an imperfect periodicity estimator capable of cue-driven movement.

\section{References}

Barnes GR, Asselman PT (1991) The mechanism of prediction in human smooth pursuit eye movements. J Physiol (Lond) 439:439-461.

Barnes GR, Donelan SF (1999) The remembered pursuit task: evidence for segregation of timing and velocity storage in predictive oculomotor control. Exp Brain Res 129:57-67.

Boman DK, Hotson JR (1988) Stimulus conditions that enhance anticipatory slow eye movements. Vision Res 28:1157-1165. 
Bon L, Lucchetti C (1991) Behavioral and motor mechanisms of dorsomedial frontal cortex of macaca monkey. Int J Neurosci 60:187-193.

Carl JR, Gellman RS (1987) Human smooth pursuit: stimulus dependent responses. J Neurophysiol 57:1446-1463.

Casini L, Macar F (1997) Effects of attention manipulation on judgments of duration and of intensity in the visual modality. Mem Cognit 25:812-818.

Collins CJ, Jahanshahi M, Barnes GR (1998) Timing variability of repetitive saccadic eye movements. Exp Brain Res 120:325-334.

Collyer CE, Church RM (1998) Interresponse intervals in continuation tapping. In: Timing of behavior (Rosenbaum DA, Collyer CE, eds), pp 6387. Cambridge, MA: MIT.

Dorris MC, Munoz DP (1995) A neural correlate for the gap effect on saccadic reaction times in monkey. J Neurophysiol 73:2558-2562.

Dorris MC, Taylor T, Klein RM, Munoz DP (1999) Influence of previous visual stimulus or saccade on saccadic reaction times in monkey. J Neurophysiol 81:2429-2436.

Gibbon J, Malapani C, Dale CL, Gallistel C (1997) Toward a neurobiology of temporal cognition: advances and challenges. Curr Opin Neurobiol 7:170-184.

Hanes DP, Schall JD (1995) Countermanding saccades in macaque. Vis Neurosci 12:929-937.

Heinen SJ, Keller EL (2003) Smooth pursuit eye movements: recent advances. In: The visual neurosciences (Chalupa LM, Werner JS, eds), pp 333-334. Cambridge, MA: MIT.

Heinen SJ, Liu M (1997) Single-neuron activity in the dorsomedial frontal cortex during smooth pursuit eye movements to predictable target motion. Vis Neurosci 14:853-865.

Heinen SJ, Badler JB, Ting W (2005) Timing and velocity randomization similarly affect anticipatory pursuit. J Vis 5:493-503

Kao GW, Morrow MJ (1994) The relationship of anticipatory smooth eye movement to smooth pursuit initiation. Vision Res 34:3027-3036.

Keller EL, Heinen SJ (1991) Generation of smooth-pursuit eye movements: neuronal mechanisms and pathways. Neurosci Res 11:79-107.

Kowler E (1989) Cognitive expectations, not habits, control anticipatory smooth oculomotor pursuit. Vision Res 29:1049-1057.

Kowler E (1990) The role of visual and cognitive processes in the control of eye movement. In: Eye movements and their role in visual and cognitive processes (Kowler E, ed), pp 1-63. Amsterdam: Elsevier.

Kowler E, Martins AJ, Pavel M (1984) The effect of expectations on slow oculomotor control-IV. Anticipatory smooth eye movements depend on prior target motions. Vision Res 24:197-210.

Krauzlis RJ, Adler SA (2001) Effects of directional expectations on motion perception and pursuit eye movements. Vis Neurosci 18:365-376.

Krauzlis RJ, Miles FA (1996) Release of fixation for pursuit and saccades in humans: evidence for shared inputs acting on different neural substrates. J Neurophysiol 76:2822-2833.

Lee K, Tehovnik EJ (1995) Topographic distribution of fixation-related units in the dorsomedial frontal cortex of the rhesus monkey. Eur J Neurosci 7:1005-1011.

Lisberger SG, Morris E, Tychsen L (1987) Visual motion processing and sensory-motor integration for smooth pursuit eye movements. Annu Rev Neurosci 10:97-129.

Logan GD, Cowan WB, Davis KA (1984) On the ability to inhibit simple and choice reaction time responses: a model and a method. J Exp Psychol Hum Percept Perform 10:276-291.

Maljkovic V, Nakayama K (1994) Priming of pop-out: I. Role of features. Mem Cognit 22:657-672.

McPeek RM, Maljkovic V, Nakayama K (1999) Saccades require focal attention and are facilitated by a short-term memory system. Vision Res 39:1555-1566.

Missal M, Heinen SJ (2001) Facilitation of smooth pursuit initiation by electrical stimulation in the supplementary eye fields. J Neurophysiol 86:2413-2425.

Missal M, Heinen SJ (2004) Supplementary eye fields stimulation facilitates anticipatory pursuit. J Neurophysiol 92:1257-1262.

Missal M, Keller EL (2002) Common inhibitory mechanism for saccades and smooth-pursuit eye movements. J Neurophysiol 88:1880-1892.

Munoz DP, Istvan P (1998) Lateral inhibitory interactions in the intermediate layers of the monkey superior colliculus. J Neurophysiol 79:1193-1209.

Ozyurt J, Colonius H, Arndt PA (2003) Countermanding saccades: evidence against independent processing of go and stop signals. Percept Psychophys 65:420-428.

Paré M, Munoz DP (1996) Saccadic reaction time in the monkey: advanced preparation of oculomotor programs is primarily responsible for express saccade occurance. J Neurophysiol 76:3666-3681.

Schall JD (1991) Neuronal activity related to visually guided saccadic eye movements in the supplementary motor area of Rhesus monkeys. J Neurophysiol 66:530-558.

Schlag J, Schlag-Rey M (1987) Evidence for a supplementary eye field. J Neurophysiol 57:179-200.

Schlag J, Schlag-Rey M, Pigarev I (1992) Supplementary eye field: influence of eye position on neural signals of fixation. Exp Brain Res 90:302-306.

Wexler M, Klam F (2001) Movement prediction and movement production. J Exp Psychol Hum Percept Perform 27:48-64.

Witney A, Goodbody S, Wolpert D (2000) Learning and decay of prediction in object manipulation. J Neurophysiol 84:334-343. 